BNL-107813-2015-IR

\title{
Safe operating conditions for NSLS-II Storage Ring Frontends commissioning
}

\author{
S. Seletskiy*, C. Amundsen, K. Ha, A. Hussain \\ *E-mail: seletskiy@bnl.gov
}

April 2015

NSLS II, Accelerator Division

Brookhaven National Laboratory

\section{U.S. Department of Energy} DOE Office of Science

Notice: This manuscript has been co-authored by employees of Brookhaven Science Associates, LLC under Contract No. DE-SC0012704 with the U.S. Department of Energy. The publisher by accepting the manuscript for publication acknowledges that the United States Government retains a non-exclusive, paid-up, irrevocable, world-wide license to publish or reproduce the published form of this manuscript, or allow others to do so, for United States Government purposes. 


\section{DISCLAIMER}

This report was prepared as an account of work sponsored by an agency of the United States Government. Neither the United States Government nor any agency thereof, nor any of their employees, nor any of their contractors, subcontractors, or their employees, makes any warranty, express or implied, or assumes any legal liability or responsibility for the accuracy, completeness, or any third party's use or the results of such use of any information, apparatus, product, or process disclosed, or represents that its use would not infringe privately owned rights. Reference herein to any specific commercial product, process, or service by trade name, trademark, manufacturer, or otherwise, does not necessarily constitute or imply its endorsement, recommendation, or favoring by the United States Government or any agency thereof or its contractors or subcontractors. The views and opinions of authors expressed herein do not necessarily state or reflect those of the United States Government or any agency thereof. 


\title{
Safe operating conditions for NSLS-II Storage Ring Frontends commissioning
}

\author{
S. Seletskiy*, C. Amundsen, K. Ha, A. Hussain \\ April 2, 2015 \\ *E-mail: seletskiy@bnl.gov
}

\section{Introduction}

The NSLS-II Storage Ring (SR) Frontends (FEs) are designed to safely accept the synchrotron radiation fan produced by respective insertion device (ID) when the electron beam orbit through the ID is locked inside the predefined Active Interlock Envelope (AIE). The Active Interlock (AI) is getting enabled at a particular beam current known as AI safe current limit. Below such current the beam orbit can be anywhere within the limits of the SR beam acceptance. During the FE commissioning the beam orbit is getting intentionally disturbed in the particular ID.

In this paper we explore safe operating conditions for the Frontends commissioning.

\section{Description of the FE}

A typical NSLS-II frontend is schematically shown in Fig. 1. It includes the following components: bending magnet photon shutter (BMPS), safety gate valve, flange absorber, fixed mask, burn-through device, set of horizontal and vertical slits, insertion device photon shutter (IDPS), another burn-through device and two additional beamline shutters.

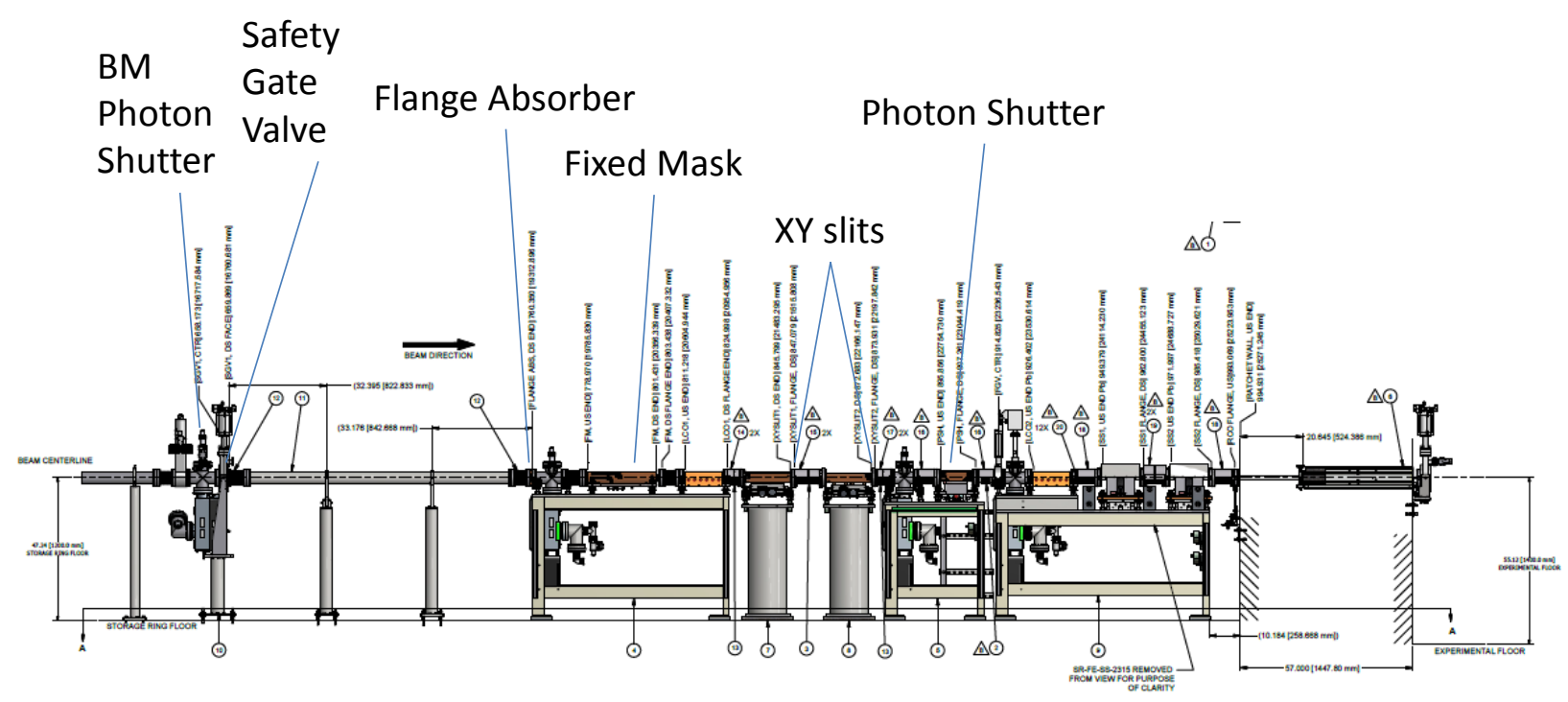

Figure 1: Typical frontend components.

Both the fixed mask and the slits can accept the full power of synchrotron radiation from the ID at maximum beam current of $500 \mathrm{~mA}$. Other FE components are designed in such way that 
when the e-beam is interlocked the possible power deposited on each component by the photon beam is negligible, i.e. that the photon beam is barely if at all touching the component. The most sensitive parts of the FE are burn-through devices, which are intentionally designed (for the goal of protecting important part of radiation shielding from melting) in such way that they vent a frontend to the atmosphere if too much power is deposited on them.

\section{AI logic}

The logic of the AI is schematically shown in Fig. 2.

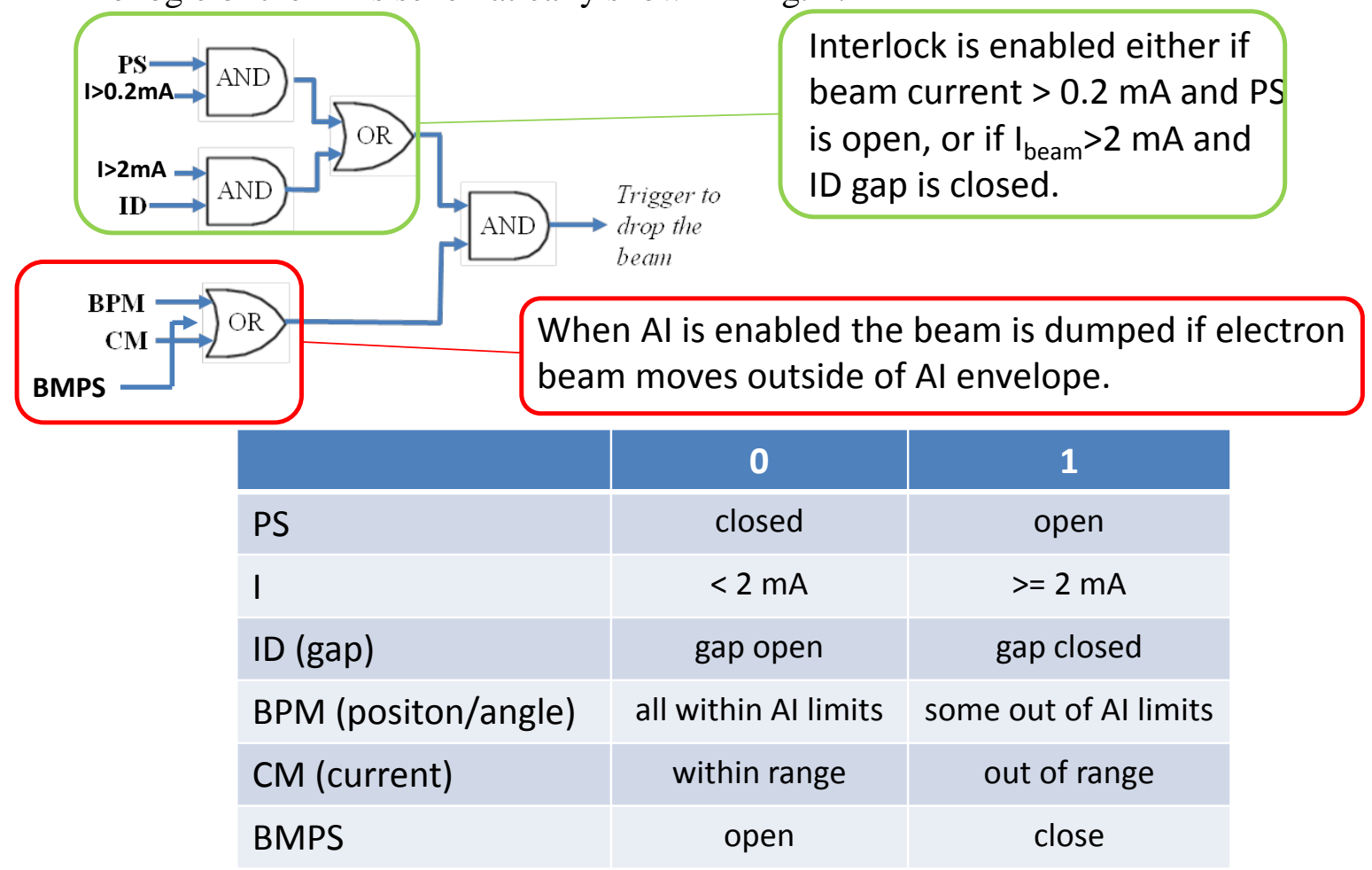

Figure 2: Logic of Active Interlock.

As Fig. 2 shows two of the FE components, the IDPS and the BMPS, are connected to the AI system. We configured active interlock in such way that the bending magnet photon shutter must stay open when the AI is enabled. In case the BMPS is getting closed, the AI drops the beam. The reason for this rule is that the BMPS was designed to take the synchrotron radiation from the bending magnet at full beam current, yet it might be damaged by the power of the ID photon beam.

Another interlocked device is IDPS; active interlock is getting enabled at $0.2 \mathrm{~mA}$ of beam current (the smallest beam current reliably readable by the SR DCCT) when the IDPS is open. Thus, we force the beam to stay inside the AIE for any beam current when the IDPS is open. This guarantees protection of all FE devices downstream of the IDPS as well as protection of all beamline components.

\section{Ray tracing}

The rough estimate for beam acceptance envelope in short and long straight sections of the storage ring is given in [1]. The actual beam acceptance is significantly smaller than the one given in [1] since [1] ignores nonlinear effects of the SR lattice, which dominate beam dynamics 
for large transverse deflections of the beam. Nonetheless, for our purpose we can use [1] as an upper limit of physically possible transverse motion of the e-beam. According to [1] in the long straight section the maximum angular deflection of the beam orbit is about 3 mrad horizontally and 1.5 mrad vertically and for short straight sections such deflections are $\sim 5$ mrad horizontally and $~ 2.5$ mrad vertically.

To determine the beam envelope for which the photon beam clears all the FE apertures (except of the fixed mask one) the ray tracing was performed for several already existing frontends. The results of ray tracing are given in Table 1.

\begin{tabular}{|l|c|c|c|c|c|}
\hline Cell & 3 & 10 & 11 & 23 & 28 \\
Beamline & HXN & IXS & CHX & CSX & XPD \\
\hline US PSH HORIZ OFFSET $(\mathrm{mm})$ & 10.00 & 10.00 & 10.00 & 10.00 & 10.00 \\
US PSH HORIZ ANGLE $(\mathrm{mr})$ & -1.00 & -1.00 & -1.00 & -1.00 & -1.00 \\
\hline US PSH HORIZ OFFSET $(\mathrm{mm})$ & -10.00 & -10.00 & -10.00 & -10.00 & -10.00 \\
\hline US PSH HORIZ ANGLE $(\mathrm{mr})$ & 1.00 & 1.00 & 1.00 & 1.00 & 1.00 \\
\hline DS PSH HORIZ OFFSET $(\mathrm{mm})$ & 1.00 & 1.70 & 1.00 & 1.30 & 3.00 \\
\hline DS PSH HORIZ ANGLE $(\mathrm{mr})$ & -1.00 & -1.00 & -1.00 & -1.00 & -1.00 \\
\hline DS PSH HORIZ OFFSET $(\mathrm{mm})$ & -1.00 & -1.70 & -1.00 & -1.30 & -3.00 \\
\hline DS PSH HORIZ ANGLE $(\mathrm{mr})$ & 1.00 & 1.00 & 1.00 & 1.00 & 1.00 \\
\hline
\end{tabular}

Table 1: Results of ray tracing for several frontends: "non-touching” e-beam envelopes.

As one can see from Table 1 the "non-touching” beam envelope is defined within +/- 1 mrad. It means that the synchrotron radiation fan from non-interlocked beam can deposit some power on the FE devices. It is worth repeating that this conclusion holds true for severely overestimated beam acceptance, in reality the power deposit for low current non-interlocked beam in the FE (with exception of the fixed mask) from ID photon beam is very low if present at all.

\section{Power density scaling}

All FE components were designed so that they can accept the synchrotron radiation power deposit from the bending magnet at full beam current. Let us consider the most sensitive parts of the FE - the burn through devices. As the analysis performed in [2] shows the burn through device can accept power density of $0.59 \mathrm{~W} / \mathrm{mm}^{2}$.

The ID producing the widest radiation fan i.e. the fan that for extreme angular deflections of e-beam can potentially "touch" the FE components (other than the fixed mask) is a damping wiggler (DW). From [3] the DW on-axis power density is $56 \mathrm{~kW} / \mathrm{mrad}^{2}$ for $0.5 \mathrm{~A}$ beam current. At $2 \mathrm{~mA}$ (the AI safe current limit) this power density becomes $224 \mathrm{~W} / \mathrm{mrad}^{2}$.

The first burn through device is located $\sim 21 \mathrm{~m}$ downstream of the DW. Therefore, the onaxis power density of the DW photon beam at the burn-through device location is $\sim 0.5 \mathrm{~W} / \mathrm{mm}^{2}$, which already is smaller than the power density the burn-through is designed to accept. Of course, the scenario of the central cone of the DW photon beam hitting the burn trough device at 90 degree angle is absolutely unphysical and possible power deposit on the FE components at 2 $\mathrm{mA}$ is much smaller than $0.5 \mathrm{~W} / \mathrm{mm}^{2}$.

\section{Conclusion}


We studied the safe operating conditions for the frontends commissioning. We described the setup of the active interlock that protects the bending magnet photon shutter from the exposure to the ID synchrotron radiation. We also explained how current AI configuration protects all FE elements downstream of the ID photon shutter as well as all beamline elements from exposure to ID radiation produced by non-interlocked e-beam. Furthermore, for the FE components upstream of the IDPS we showed that even under extremely unrealistic assumptions about beam orbit deflections in the IDs the most vulnerable part of the FE does not see the power density higher than what it is designed to accept.

We conclude that current AI configuration is safe for both FE commissioning and routine operations. This conclusion is corroborated by our experience with commissioning the first 8 frontends at NSLS-II SR. Indeed, during the FE commissioning (at beam current $<2 \mathrm{~mA}$ ) we didn't see any temperature rise in the FEs, neither we observed any significant vacuum activity in the frontends.

\section{References}

[1] I. Pinayev, Orbit Envelopes for NSLS-II Ring Synchrotron Radiation Sources.

[2] A. Hussein, FE Burn-Thru Devices FEA, February 28, 2014.

[3] Andy Broadbent, RSI for the Front-Ends for the Six NSLS-II Project Beamlines, 17 Dec 2010. 